\title{
DIRECTIONAL SOLIDIFICATION OF A BINARY ALLOY INTO A CELLULAR CONVECTIVE FLOW: LOCALIZED MORPHOLOGIES
}

Y.-J. Chen and S. H. Davis, Department of Engineering Sciences and Applied Mathematics, Northwestern

University, Evanston, IL60208

\begin{abstract}
A stcady, two dimensional cellular convection modifies the morphological instability of a binary alloy that undergoes directional solidification. When the convection wavelength is far longer than that of the morphological cells, the bchavior of the moving front is described by a slow, spatial-temporal dynamics obtained through a multiple-scale analysis. The resulting system has a 'parametric-excitation' structure in space, with complex parameters characterizing the interactions between flow, solute diffusion, and rejection. The convection stabilizes two dimensional disturbances oriented with the flow, but destabilizes three dimensional disturbances in general. When the flow is weak, the morphological instability behaves incommensurably to the flow wavelength, but becomes quantized and forced to fit into the flowbox as the flow gets stronger. At large flow magnitudes the instability is localized, confined in narrow envelopes with cells traveling with the flow. In this case the solutions are discrete eigenstates in an unbounded space. Their stability boundary and asymptotics are obtained by the WKB analysis.
\end{abstract}

\section{INTRODUCTION}

In the absence of flow, the morphological instability of a binary alloy undergoing directional solidification is driven by the adverse gradient of solute concentration at the solid-liquid interface. This instability occurs when the interface advances with a speed $I$ greater than a critical value $T_{c}$, when cellular patterns form on the moving front. When the liquid (melt) is flowing, the solute concentration profilc is altered. In general, the liquid motions may delay or promote the instability depending on the interactions between the solute and momentum transport.

Flow-modified morphological instability has been studied by many authors (e.g. see [6] for a review). Flows can be inherent in the nature of the solidifying process, such as solutal convection in a density stratified melt ([3], [4]). The motion here is due to buoyancy effects that exist even when the interface is not deformable. Flows can also be imposed through a far-field pressure gradient. In this case the purpose of the flows may be in the interest of material processing. The examples include plane Couette flow [5], asymptotic suction profile ([7], [8], [11]), and stagnation-point flow [1].

In the present analysis we examine the morphological instability of a pre-existing cellular convective flow (see figure 1). Such flows exist when hydrodynamic instabilities occur before the morphological instability, or when the liquid is subjected to a high-frequency vibration or accelcration (g-jitter). The flow is considered to be spatially periodic, with a wavelength $2 \pi / \alpha$ typically much longer than the wavelength $2 \pi / 3$ of the morphological instability. This assumption is appropriate for metallic alloys for which typical Schmidt numbers are large, and the viscous length-scale can be ten or hundred times longer than the diffusion length-scale. The present work is related to that of Bühler \& Davis [2], in which numerical calculations to the linear problem have been performed. Their study showed that in such flows the morphological instability can be confined in localized, stationary envelopes distributed periodically on the interface. Each envelope contains many morphological cells traveling in the flow direction. The stability and mechanism of the onset of this solution have yet to be revealed.

We treat the solutal-momentum transport as a perturbation of the Mullins-Serkerka problem [10]. The 'pure' (no-flow) morphological instability has its critical wavenumber $\beta_{c}$ at a critical morphological number $M_{c}$, as shown schematically in figure $2 \mathrm{a}$. For the smallness of $\alpha$ comparing with $\beta_{c}$, we evoke a multiple-scale analysis near the critical point $\left(\beta_{c}, M_{c}\right)$. The resulting dynamics has a 'parametric-excitation' form in the slow, spatial variable, where the periodic coefficients are generated by the cellular flow. The linear problem is then solved by the numerical branch-tracing technique with the asymptotic structures obtained through a WKB-type analysis.

We observe that disturbances (rolls) perpendicular to the flow (figure $2 b$ ) are stabilized, since the tangential component of the flow either compresses or stretches the morphological cells along the interface. That is, a wave structure which is unstable without the flow has now been altered and pushed to the regime where the surface tension or solute diffusion help to stabilize. In contrast, rolls aligned with the flow (figure $2 c$ ) are desta- 


\section{BASIC STATE}

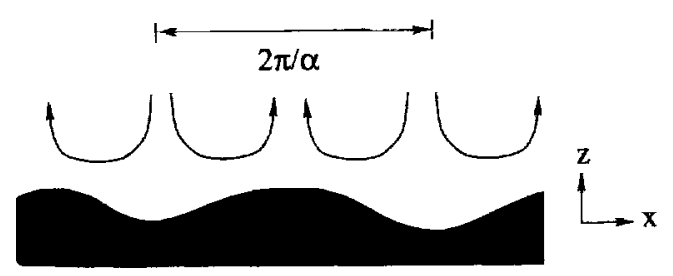

Figure 1: Schematic diagram for a spatially periodic flow impressing on the solid-liquid interface of a binary alloy.

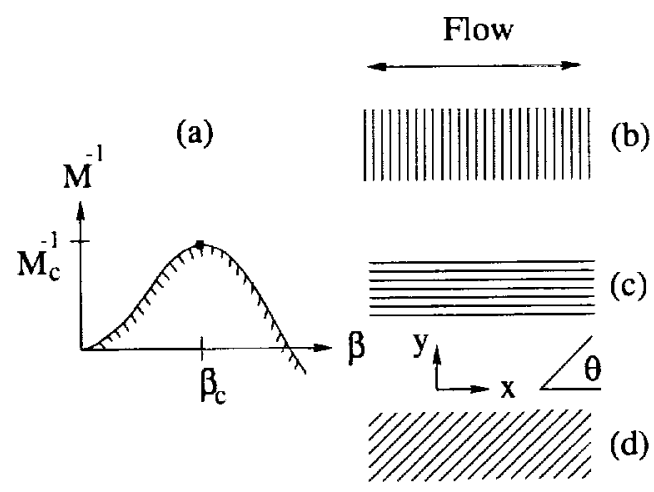

Figure 2: (a) Marginal stability diagram of the pure morphological instability. Solutions in hatched area arc unstablc. The morphological cell has a wave-vector (b) parallel, (c) perpendicular, and (d) an angle $\theta$ to the flow.

bilized by the normal flow component. The morphologies have incommensurate structures (aperiodic in space) when the flow is weak, and develop localized wave envelopes, as observed in [2], when the flow strength increases. The stability boundary and the onset of the traveling cells are identified.

\section{Solutal Transport Equations}

Consider a dilute binary alloy solidifying into a cellular flow field. The averaged solid-liquid interface moves in a constant velocity $V$. The solute rejected on the interface has a partition coefficient $k$ and diffusivity $D$ in the liquid. We invoke the 'frozen temperature approximation' [9] that gives the temperature $T$ in the solid and liquid permanently by $T=T_{0}+G z$, where $G$ is the imposed temperature gradient, and $T_{0}$ is a reference temperature. The coordinate variable $z$ is fixed on the moving front with positive $z$-direction pointing to the liquid. The interfacial coordinates are spanned by the $(x, y)$-axes. The temperature will not be disturbed when the thermal boundary layer is far thicker than the momentum and concentration boundary layers.

We choose the velocity, time, length, and concentration scales to be $V, D / T^{2}, \ell_{c}=D / V$, and $\Delta c=$ $(1 / k-1) c_{\infty}$, respectively. Referred to those scalings and chosen coordinates, the solutal transport equation in the liquid can be written

$$
\begin{gathered}
S c^{-1}\left\{\partial_{t} v+\left(v-\hat{e}_{z}\right) \cdot \nabla v\right\}=-\nabla p+\nabla^{2} v, \\
\nabla \cdot v=0, \quad \partial_{t} c+\left(v-\hat{e}_{z}\right) \cdot \nabla c=\nabla^{2} c .
\end{gathered}
$$

In the above formulation we have assumed the melt to be an incompressible, Newtonian liquid. The Schmidt number $S c=\nu / D$ measures the strength of liquid viscosity to the solute diffusivity.

On the solid-liquid interface $z=h(x, y, t)$, the noslip condition $v=0$ is applied. It is also assumed that the density change upon solidification is negligible, and the velocity $v$ approaches the far-ficld distribution $v_{\infty}$ as $z \rightarrow \infty$. The ficld $v_{\infty}$ is $\frac{2 \pi}{\Omega}$ periodic in $x$ and is the driving force of the convective flow. The solute boundary conditions on the interface consist of the mass conservation law and a local thermodynamic equilibrium:

$$
\begin{gathered}
v_{n}\{(1-k) c+k\}=-\hat{n} \cdot \nabla c \\
M^{-1} h=1-c+2 \Gamma k(h) .
\end{gathered}
$$

Here, $\hat{n}$ is the unit normal vector pointing into the liquid phase, $v_{n}$ the speed of the front normal to itself, and $k$ : the mean curvature, a functional of the interface shape function $h$. The mass conservation law (2) assumes that solute diffusion in the solid is negligible comparing with that in the liquid. The Gibbs-Thomson condition (3) depicts the alteration of temperature of interface from the equilibrium melting temperature of the pure solvent due to the presence of solute (constitutional undercooling) and the curvature of the interface itself (capillary undercooling). The morphological number and surface encrgy parameter are given by

$$
M=\frac{m G_{c}}{G}, \quad \Gamma=\frac{T_{m} \gamma}{m G_{c} \ell_{c}^{2} L_{v}}
$$

where $G_{c}=-\Delta c / \ell_{c}$ measures the concentration gradient. The liquidus slope of the phase diagram is denoted by $m$, and the parameters $\gamma, T_{m}, L_{v}$ stand for surface tension, solvent melting temperature, and latent heat per unit volume, respectively.

\section{Basic State}

The given cellular flow has a spatial period $\frac{2 \pi}{a}$, which is considered to be much longer than the morphologi- 


\section{DYNAMICS IN SLOW VARIABLES}

cal length-scale $\ell_{c}$. The velocity field is represented by a simple sinusoidal function in the direction $x$, and decays to zero at the interface exponentially. We use $P$ to denote the value of the far-ficld velocity. Its tangential component is written

$$
u=-\left\{1-\mathrm{e}^{(h-z) / s}\right\} P \sin \alpha x
$$

The parameter $s$ distinguishes a general class of flows [2]. Its magnitude represents the thickness of the viscous boundary layer near the interface. Flow type in (4) is motivated by the 'asymptotic suction profile', in which the remote ficld, $P \sin \alpha x$, is replaced by a constant, and the parameter $s$ is equivalent to the Schmidt number $S c$. The asymptotic suction profile is an exact solution to the momenlum equation, while the remote flow (4) serves as an approximation for $\alpha \ll 1$ and flows near the interface. This enables us to simplify the analysis and consider only the situation of large Schmidtnumber, metallic alloys $(S c \rightarrow \infty)$. Consequently, the liquid inertia is neglected, and the parameter $s$ can takc any positive value $(0<s<\infty)$.

Flows with $s \leq 1$ can occur in a Hele-Shaw-like apparatus for example, where the close proximity of the sidewalls creates a thin viscous boundary layer. Boundary layers of exponential type are also observed in electrically conducting liquid with flows induced by strong magnetic ficlds. For those examples the spatial period $\frac{2 \pi}{n}$ can be introduced through solutal buoyancy or magnetohydrodynamic instability.

While the flow field is treatcd as an approximate solution to the momentum transport equation, the resulting solutal and interfacial profiles $\left(c_{0}, h_{0}\right)$ can be obtained by introducing the expansions

$$
\begin{aligned}
c_{0}(\alpha x, z) & \sim \mathrm{e}^{h_{0}-z}+\alpha \delta f_{1}(z) \cos \alpha x \\
h_{0}(\alpha x) & \sim 0+\alpha \delta f_{2}(z) \cos \alpha x
\end{aligned}
$$

into the solutal transport equation and boundary conditions. Functions $f_{1}$ and $f_{2}$ can be obtained in a straightforward manner. In (5) we have rescaled the flow effects and employed a expansion parameter $\alpha \delta[2]$, where

$$
\delta \equiv \frac{P}{1+s},
$$

to indicate the order of magnitude of the flow perturbations. The expansion uses that fact that the solutal and interfacial shape functions are weakly perturbed off the profiles of the quiescent case as $\alpha \delta \ll 1$. The parameter $\delta$ here represents the magnitude of velocity near the interface when $s \ll 1$, and the velocity gradient when $s \gg 1$. The scaling is introduced in the expansions, since only the magnitude of the flow is important when the concentration boundary layer is subjected to an extremely thin viscous layer, while for a thick viscous layer only the velocity gradient near the interface is relevant to the convective transport.

\section{Dynamics In Slow Variables}

The imposed flow field breaks the rotational symmetry of the plane surface. We shall subject the basic state $(4,5)$ to a 'roll-like' disturbance, with an angle $\theta$ to the flow direction (figure $2 \mathrm{~d}$ ). Recall that, in the absence of flow, the most dangerous perturbation is the normal mode $\mathrm{e}^{i \vec{\beta}_{c} \cdot(x, y)}$ at the critical morphological number $M_{c}$. In the case with flow the motion introduces a slow, spatial change. It is thus reasonable to assume that the most dangerous disturbance now has a form $f(\epsilon, r) \mathrm{e}^{i \vec{\beta}_{c} \cdot(x, y)}$, $\epsilon \ll 1$, where $f(\epsilon x)$ is a slowly varying function. The parameter $f$ enables us to perform a multiple-scalc analysis near the point of $\left(\beta_{c}, M_{c}\right)$ (sec figure 2).

Formally, we assume a perturbation expansion:

$$
\begin{gathered}
M^{-1} \mapsto M_{c}^{-1}-\mu_{1} \epsilon^{2} m, \quad \alpha \mapsto \epsilon \alpha, \quad \delta \mapsto \mu_{2} \epsilon^{2} \hat{\delta}, \\
\tau=\mu_{3} \epsilon^{2} t, \quad \eta=\epsilon x, \quad \xi=\epsilon^{2} x \\
u \sim \boldsymbol{u}_{0}+\left\{\epsilon \boldsymbol{u}_{1}(\tau, \eta, \xi, z)+\epsilon^{2} u_{2}+\right. \\
\left.\epsilon^{3} \boldsymbol{u}_{3}\right\} \mathrm{e}^{i \overline{3}_{c} \cdot(\boldsymbol{x}, y)}+\{\text { c.c. }\}
\end{gathered}
$$

where $u=(c, h),\{c . c$.$\} represents complex conjugate,$ and $\mu_{i}$ are coefficients which will be determined later to renormalize the slow-variable dynamics. By substituting the above expansions into the governing transport equations, and collecting the like powers of $\epsilon$, we arrive at a series of linear problems, which can be written as

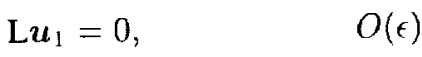

$$
\begin{aligned}
& \mathbf{L} \boldsymbol{u}_{2}=F_{1}\left(\boldsymbol{u}_{1}\right), \quad O\left(\boldsymbol{\epsilon}^{2}\right) \\
& \mathbf{L} \boldsymbol{u}_{3}=F_{2}\left(\boldsymbol{u}_{1}, \boldsymbol{u}_{2}\right), \quad O\left(\epsilon^{3}\right),
\end{aligned}
$$

where $L$ is the linear operator of the no-flow case. Since the operator $\mathrm{L}$ is singular, the solvability condition is necessary to solve $u_{i}$ at each order. The quadratic nature of the turning point at $\left(\beta_{c}, M_{c}\right)$ results in the inhomogencous term $F_{1}\left(u_{1}\right)$ that is already in the range of $\mathrm{L}$. The leading-order behavior of $\left(c_{1}, h_{1}\right)$ is then determined by the solvability condition at $O\left(\epsilon^{3}\right)$. This procedure results in a dynamical system in the slow variable (dropping subscript ' 1 '):

$$
\begin{array}{r}
\partial_{\tau} h=\{m+i \hat{\delta} \cos \theta \sin \alpha \eta\} h+\left\{\cos \theta \partial_{\eta}\right\}^{2} h- \\
a h|h|^{2}+O(\epsilon \alpha \hat{\delta} \cos \alpha \eta h),
\end{array}
$$




\section{LINEAR STABILITY ANALYSIS}

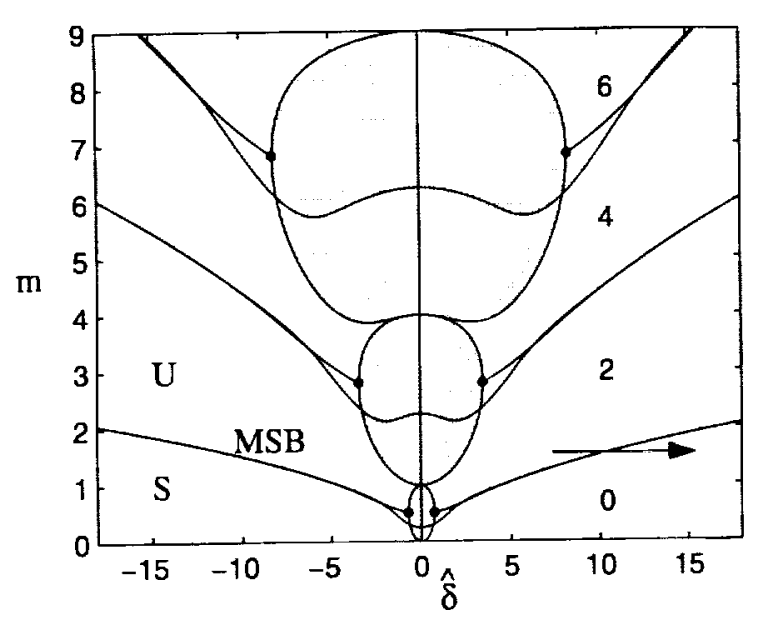

Figure 3: Characteristic curves for the 2d-roll equation. Shaded area represents solutions that are incommensurate with the flow. Solid circles are double degenerate points. The arrow indicates a path showing flowinduced stability. Symbol ' $S$ ' denotes stability, ' $U$ ' denotes instability, and 'MSB' denotes marginal stability boundary.

with appropriate constants $\mu_{i}$. The Landau constant $a$ determines the supercriticality $(a>0)$ or subcriticality $(a<0)$ of the system. When $\hat{\delta} \equiv 0$, equation(6) recovers the system of the pure morphological problem in [12]. Equation(6) is written in the form for which the morphological instability has an $O(1)$ wavenumber in the remote-flow direction ( $\cos \theta=O(1)$, cf. figure $2 \mathrm{~b}$ ). In this case the flow component $\hat{\delta} \sin \alpha \eta$ tangential to the interface has the leading-order contribution, and the normal component $\alpha \hat{\delta} \cos \alpha \eta$ is formally $O(\epsilon)$. That is, not until very close to the stagnation points $\left(\eta=\frac{n \pi}{\alpha}\right)$ does the effect of the normal component enter.

When one rotates the wave-vector of the morphological cells to be nearly perpendicular to the flow direction, $(\cos \theta=O(\epsilon)$, cf. figure $2 \mathrm{c})$, the tangential and normal flow components will have the same orders of magnitude; equation(6) will then need to be modified. In this regime we rescale the flow parameter $\delta \mapsto \mu_{4} \epsilon \delta^{*}$ such that the flow interacts with the morphological instability at $O\left(\epsilon^{3}\right)$. The slow-variable dynamics is then rewritten

$$
\begin{array}{r}
\partial_{\tau} h=\left\{m+\delta^{*} \sin \alpha \eta \partial_{\eta}+\chi \alpha \delta^{*} \cos \alpha \eta\right\} h+ \\
\epsilon^{2} \partial_{\eta}^{2} h-a h|h|^{2}
\end{array}
$$

where $\chi=\chi(k, \Gamma, s)$ is an $O(1)$ parameter. Equa-

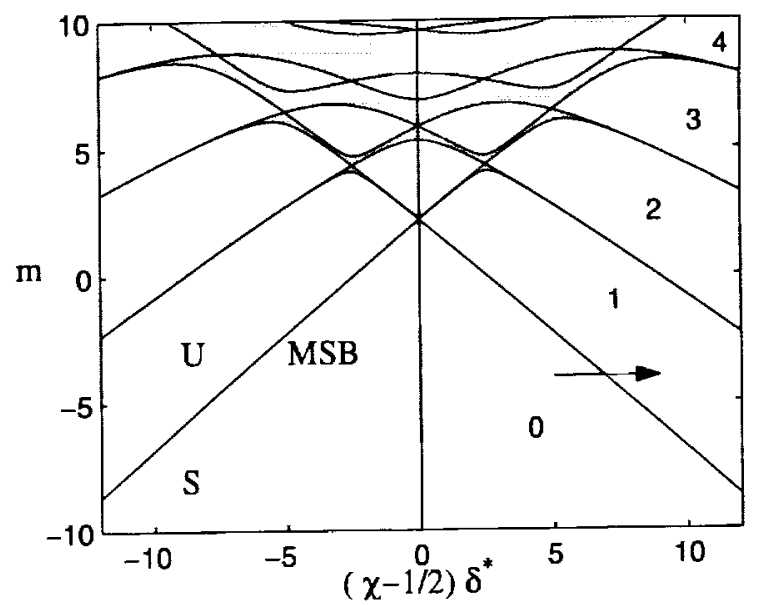

Figure 4: Characteristic curves for the longitudinal-roll equation. Shaded area represents solutions that are spatially incommensurate with the flow. The arrow indicates a path showing flow-induced instability. Symbol ' $S$ ' denotes stability, 'U' denotes instability, and 'MSB' denotes marginal stability boundary.

tion (7) is a singular perturbation problem with the surface tension force $\epsilon^{2} \partial_{\eta}^{2} h$ now acting as a regularization term.

\section{Linear Stability analysis}

To determine the initial behavior of small disturbances we neglect the nonlinear terms in $(6,7)$. It is convenient in the analysis to study only the normal mode: $h(\tau, \eta) \mapsto \mathrm{e}^{i \omega \tau} h(\eta)$, and have the disturbance wavevector oriented either with $x$ or $y$ axis (cf. figure $2 b$, c). The deduced system are then referred to as ' $2 \mathrm{~d}$ ' and 'longitudinal' (3d) rolls respectively, according to their relevant morphological structures:

$$
\begin{array}{r}
\text { 2d roll: }\{m-i \omega+i \hat{\delta} \sin \alpha \eta\} h+\partial_{\eta}^{2} h=0, \\
\text { longitudinal roll: }\left\{m-i \omega+\delta^{*} \sin \alpha \eta \partial_{\eta}+\right. \\
\left.\chi^{\alpha} \delta^{*} \cos \alpha \eta\right\} h+\epsilon^{2} \partial_{\eta}^{2} h=0 .
\end{array}
$$

As noted, the longitudinal-roll equation is a singular perturbation problem; thus the term $\epsilon^{2} \partial_{\eta}^{2} h$ must be retained. The systems have parametric-excitation form, driven by the imposed flow. In analogy to a pendulum problem, the two 'spatial pendula' here have periodic spring-constants in accordance with the flow period, controlled by complex parameters. The Floquet theory describes the complicated nature of the spatial behaviors. The coefficients have period $\frac{2 \pi}{\alpha}$, and there 
are solutions in the form

$$
h(\eta)=\mathrm{e}^{\mu \eta} \phi(\eta), \quad \phi(\eta+2 \pi / \alpha)=\phi(\eta)
$$

Since we are concerned only with the spatially bounded solutions (eigenfunctions); this demands $\operatorname{Re} \mu=0$. Figures $3 \& 4$ plot the boundaries of those solutions, in which the lines were obtained by the numerical branchtracing technique, and numbers labelled represent the number of unstable modes ( $\operatorname{Im} \omega<0$ ) that have lengths fitted into the flow-box. The branching solutions were traced in the $(\delta, m, \omega)$-space, while only period $\frac{2 \pi}{a}$ and $\frac{4 \pi}{a}$ solutions are plotted. Solutions within the boundarics are aperiodic functions, and the interfacial perturbations are incommensurate (spatially unsynchronized) with the flow. As the flow rate $|\delta|$ increases, the 2 d-roll solution (figure 3 ) changes from stationary ( $\omega \equiv 0$ ) to time-dependent modes at the double degenerate points (solid circles), where branches of solutions collide and pairs of oscillatory modes $\left(\omega= \pm \omega^{*}\right)$ bifurcate. In conIrast, all solutions of the longitudinal roll are stationary (figure 4), with the 'incommensurate bands' shrinking to thin, film-like layers as $\epsilon \rightarrow 0^{+}$. This behavior suggests that the eigenstates are essentially quantized. That is, discretc eigenmodes exist in an unbounded state, and the induced morphology has a structure spatially synchronizing with the flow-periodicity. In the longitudinalroll perturbation, this quantization occurs due to the regularization of the surface tension force (cf. equation 9). The morphological cells are aligned with the flow, with a secondary structure near the flow-stagnation points (figure 5). For the $2 \mathrm{~d}$ disturbance, the quantization happens at high flow rates after the double degenerate points. The oscillatory modes are in agreement with the travelingcell solutions observed in [2], in which cells are traveling in the flow direction, with the amplitude confined in narrow envelopes located between the stagnation points (figure 6).

Our analysis also indicates that the flow stabilizes the $2 \mathrm{~d}$ disturbance since the marginal morphological number $m_{c}$ has been delayed $\left(m_{c}>0\right)$, but destabilizes the $3 \mathrm{~d}$ disturbance with the instability occurs before the pure morphological instability $\left(m_{c}<0\right)$. A WKB type analysis delivers the asymptotics of the stability boundaries and the local structures:

$$
\begin{gathered}
\text { 2d-roll: } \quad m_{c} \propto|\alpha||\hat{\delta}|^{1 / 2}, \omega \propto \hat{\delta} \\
h \sim \mathrm{e}^{-\mu_{5}|\alpha||\hat{\delta}|^{1 / 2}\left(\eta-\eta_{0}\right)^{2}} .
\end{gathered}
$$

longitudinal-roll: $m_{c} \times-\left|\alpha \delta^{*}\right|, \quad \omega \equiv 0$,

$$
h \sim \mathrm{e}^{-\mu_{0}\left|\alpha \delta^{*} / \epsilon^{2}\right|\left(\eta-\eta_{0}\right)^{2}} .
$$

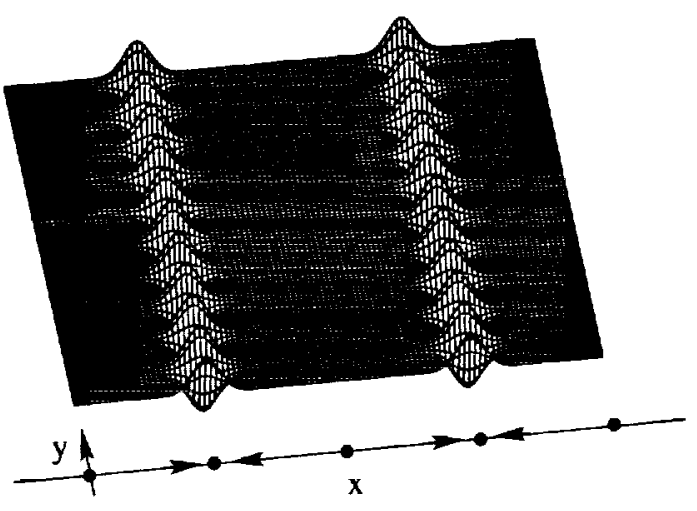

Figure 5: Longitudinal-roll morphology (perspective view) Solid circles are stagnation points, and arrows the flow direction.

(a)

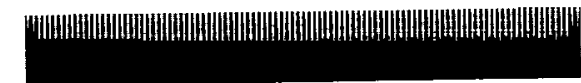

(b)

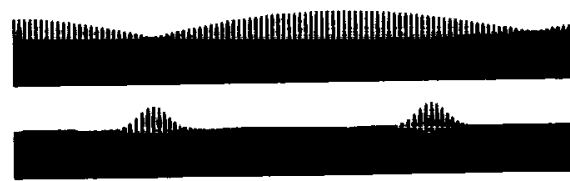

(c)

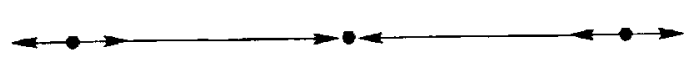

Figure 6: Two dimensional rolls (side-view) predicted by the lincar stability analysis: (a) purc, (b) incommensurate, and (c) localized morphologics. Solid circles are stagnation points, and arrows the flow direction.

In agreement with the results of the branch-tracing calculation, the eigenmodes have localized structures at the positions where, for the $2 \mathrm{~d}$, the tangential flow component is the strongest and, for the $3 \mathrm{~d}$, the normal flow component dominates. The discrete modes are the 'turningpoint' solutions in the WKB analysis.

\section{Discussion AND SummaRy}

Linear stability analysis shows the flow stabilizes $2 \mathrm{~d}$ but destabilizes $3 \mathrm{~d}$ disturbances. The mechanism may be tightly bound with the structure of the marginal stability curve shown in figure $2 \mathrm{a}$. A disturbance along the flow direction (figure 6) is compressed or stretched near the stagnation points by the tangential flow component. This changes its wavenumber so that the local $\beta$ becomes larger or smaller than the critical value for the pure morphological instability. The surface ten- 


\section{REFERENCES}

sion force or solute diffusion help stabilize the interface locally. However, when a disturbance has $3 d$ structure (figure 5), the normal flow component will compress the concentration boundary layer at the stagnation points and promotes the constitutional undercooling. This effectively lowers the morphological number such that the interface is locally destabilized. These two mechanisms predict that, near the onset of the instability, patterns like figure 6 may occur in a confined, $2 \mathrm{~d}$ geometry (Hele-Shaw slot, for example). In a fully three dimensional setting one would expect to see a pattern close to figure 5 , since it is the more unstable.

In summary, we investigate the effects of a cellular convective flow on the directional solidification of a dilute binary alloy. The imposed flow has spatial wavenumber $\alpha$ and strength parameter $\delta$ that controls the perturbation to the interfacial deformation. A multiple-scale analysis is performed near the onset of the morphological instability, which results in a weakly nonlinear equation in slow, space and time variables. The dynamics of the front is parametrically excited by the imposed flow. Its linear problem is solved by the numerical branchtracing method. It is found that the remote, spatially periodic flow stabilizes $2 \mathrm{~d}$ disturbances in the flow direction, yet promotes $3 \mathrm{~d}$ instability. The localized morphological instability previously observed are identified as the quantization of the eigenstates, in which the unstable modes are discrete in an unbounded space, and the perturbed interfacial structures are forced to be spatially synchronized with the flow. The stability boundary and the asymptotic structure of the eigenmodes are obtained by the WKB method in the limit of $\alpha \rightarrow 0^{+}$.

\section{ACKNOWLEDGMENT}

This work was supported by NASA, Microgravity Science and Application Program.

\section{REFERENCES}

[1] Brattkus, K. \& Davis, S. H. J. Cryst. Growth, 89 , $423,1988$.

[2] Bühler, L. \& Davis, S. H. J. Cryst. Growth, (In press) 1998.

[3] Coriell, S. R., Cordes, M. R., Boettinger, W.S. \& Serkerka, R. F. J. Cryst. Growth, 49, 13, 1980.

[4] Coriell, S. R., \& McFadden, G.B. J. Cryst. Growth, 94, 513,1989.

[5] Coriell, S.R., McFadden, G.B. \& Boisvert, R.F. J. Cryst. Growth, 69, 15, 1984.

[6] Davis., S.H., in: Handbook of Crystal Growth 1, Hurle, D.T.J. editor, vol. 1 ch. 13, p861. Elscvier Science Publishers, 1993.

[7] Forth, S.A. \& Wheeler, A. A. J. Fluid Mech., 202, $339,1989$.

[8] Hobbs, A. K. \& Metzener, P. J. Cryst. Growth, 112, 539, 1991.

[9] Langer, J. S. Rev. Mod. Phys., 52(1), 1, 1980.

[10] Mullins, W.W. \& Serkerka, R.F. J. Appl. Phys., 35(2),444, 1964 .

[11] Schulze, T. P. \& Davis, S. H. J. Cryst. Growth, 143, 317, 1994.

[12] Wollkind, D. J. \& Segel, L. A. Philos. Trans. Roy. Soc. London A 268, 351, 1970. 